\title{
THE ALEXANDER POLYNOMIAL OF A 3-MANIFOLD AND THE THURSTON NORM ON COHOMOLOGY
}

\author{
By CURTIS T. MCMULLEN
}

ABSTRACT. - Let $M$ be a connected, compact, orientable 3-manifold with $b_{1}(M)>1$, whose boundary (if any) is a union of tori. Our main result is the inequality

$$
\|\phi\|_{A} \leqslant\|\phi\|_{T}
$$

between the Alexander norm on $H^{1}(M, \mathbb{Z})$, defined in terms of the Alexander polynomial, and the Thurston norm, defined in terms of the Euler characteristic of embedded surfaces. (A similar result holds when $b_{1}(M)=1$.) Using this inequality we determine the Thurston norm for most links with 9 or fewer crossings.

(C) 2002 Éditions scientifiques et médicales Elsevier SAS

RÉSUMÉ. - Soit $M$ une variété de dimension 3 connexe et compacte, telle que $b_{1}(M)>1$, et dont le bord (s'il y en a un) est une réunion de tores. Notre résultat principal est l'inégalité

$$
\|\phi\|_{A} \leqslant\|\phi\|_{T}
$$

entre la norme d'Alexander sur $H^{1}(M, \mathbb{Z})$, définie à partir du polynôme d'Alexander, et la norme de Thurston, définie à partir de la caractéristique d'Euler des surfaces plongées. (On dispose d'un résultat similaire lorsque $b_{1}(M)=1$.) À l'aide de cette inégalité, nous déterminons la norme de Thurston pour la plupart des entrelacs avec au plus 9 croisements.

(c) 2002 Éditions scientifiques et médicales Elsevier SAS

\section{Introduction}

Let $M$ be a connected, compact, orientable 3-manifold whose boundary (if any) is a union of tori. In this paper we study the Alexander norm on $H^{1}(M, \mathbb{Z})$, defined by

$$
\|\phi\|_{A}=\sup \phi\left(g_{i}-g_{j}\right)
$$

where $\Delta_{M}=\sum a_{i} g_{i}$ is the Alexander polynomial of $M$.

For manifolds with $b_{1}(M) \geqslant 2$ our main result is the inequality

$$
\|\phi\|_{A} \leqslant\|\phi\|_{T},
$$

where $\|\phi\|_{T}$ is the Thurston norm (measuring the minimal complexity of an embedded surface dual to $\phi)$. The inequality (1.1) generalizes the classical relation $\operatorname{deg} \Delta_{K}(t) \leqslant 2 g(K)$ for knots.

\footnotetext{
Research partially supported by the NSF.
} 
Although the Thurston norm has been calculated in particular examples, few are documented in the literature. In Section 7 we use (1.1) to systematically determine the Thurston norm for most links with 9 or fewer crossings (128 of the 131 in Rolfsen's tables). To facilitate this computation, in the Appendix we provide a table of links with homeomorphic complements.

We now turn to a detailed statement of the main result, give a sketch of the proof and formulate open questions.

The Alexander norm. Let $G$ be a finitely-generated group. The maximal free abelian quotient of $G$ will be denoted by

$$
\operatorname{ab}(G)=H_{1}(G, \mathbb{Z}) /(\text { torsion }) \cong \mathbb{Z}^{b_{1}(G)},
$$

where $b_{1}(G)=\operatorname{dim} H_{1}(G, \mathbb{Q})$ is the first Betti number of $G$. Let

$$
\Delta_{G}=\sum_{1}^{N} a_{i} g_{i} \in \mathbb{Z}[\operatorname{ab}(G)]
$$

be the Alexander polynomial of $G$ (defined in Section 2). Assume the coefficients $a_{i}$ are nonzero and the group elements $g_{i}$ are distinct.

We define the Alexander norm on $H^{1}(G, \mathbb{Z})=\operatorname{Hom}(G, \mathbb{Z})$ by

$$
\|\phi\|_{A}=\sup _{i, j} \phi\left(g_{i}-g_{j}\right) .
$$

The unit ball of the Alexander norm is, up to scale, the dual of the Newton polytope of the Alexander polynomial. By convention $\|\phi\|_{A}=0$ if $\Delta_{G}=0$.

The Alexander norm on $H^{1}(M, \mathbb{Z})$ is defined by setting $G=\pi_{1}(M)$ and using the isomorphism $H^{1}(M, \mathbb{Z})=H^{1}(G, \mathbb{Z})$.

The Thurston norm. For any compact surface $S=S_{1} \sqcup S_{2} \sqcup \cdots \sqcup S_{n}$, let $\chi_{-}(S) \geqslant 0$ be the sum of $\left|\chi\left(S_{i}\right)\right|$ over all components of $S$ with negative Euler characteristic. The Thurston norm on $H^{1}(M, \mathbb{Z})$ is defined by:

$$
\begin{aligned}
\|\phi\|_{T}=\inf \left\{\chi_{-}(S):\right. & (S, \partial S) \subset(M, \partial M) \text { is an oriented embedded surface, } \\
& \text { and } \left.[S] \in H_{2}(M, \partial M) \text { is dual to } \phi\right\} .
\end{aligned}
$$

The Alexander and Thurston norms are sometimes degenerate (they can vanish on nonzero vectors).

THEOREM 1.1 (Comparison of norms). - Let $M$ be a compact, connected, orientable 3-manifold whose boundary (if any) is a union of tori. Then the Alexander and Thurston norms on $H^{1}(M, \mathbb{Z})$ satisfy

$$
\|\phi\|_{A} \leqslant\|\phi\|_{T}+ \begin{cases}0 & \text { if } b_{1}(M) \geqslant 2, \\ 1+b_{3}(M) & \text { if } b_{1}(M)=1 \text { and } H^{1}(M, \mathbb{Z})=\mathbb{Z} \phi .\end{cases}
$$

Equality holds if $\phi: \pi_{1}(M) \rightarrow \mathbb{Z}$ is represented by a fibration $M \rightarrow S^{1}$ with fibers of non-positive Euler characteristic.

Here $b_{i}(M)=\operatorname{dim} H_{i}(M, \mathbb{Q})$ is the $i$ th Betti number of $M$. 
Sketch of the proof. The proof depends on a determination of the Alexander ideal of a 3-manifold. Let

We will show:

$$
p(M)= \begin{cases}0 & \text { if } b_{1}(M) \leqslant 1 \\ 1+b_{3}(M) & \text { otherwise }\end{cases}
$$

1. The Alexander ideal of $G=\pi_{1}(M)$ satisfies

$$
I(G)=m^{p(M)} \cdot\left(\Delta_{G}\right),
$$

where $m=m(\operatorname{ab}(G))$ is the augmentation ideal, and $\left(\Delta_{G}\right)$ is the principal ideal generated by the Alexander polynomial.

2. Assume $\Delta_{G} \neq 0$. Then for primitive $\phi \in H^{1}(M, \mathbb{Z})$, we have

$$
b_{1}(\operatorname{Ker} \phi)=\operatorname{deg} \Delta_{G}\left(s^{\phi}\right)+p(M)=\|\phi\|_{A}+p(M),
$$

so long as $\phi$ lies in the cone on the open faces of the Alexander norm ball.

3. Let $S \subset M$ be an embedded surface dual to $\phi$; then

$$
b_{1}(S) \geqslant b_{1}(\operatorname{Ker} \phi)
$$

4. Combining these inequalities gives

$$
b_{1}(S)-p(M) \geqslant\|\phi\|_{A},
$$

and the comparison with the Thurston norm follows by relating $|\chi(S)|$ and $b_{1}(S)$.

In Sections 2-4 we discuss the Alexander invariants of a general group, and their relationship to cohomology and $b_{1}$ of cyclic covers. The structure of the Alexander ideal of a 3-manifold is determined in Section 5. In Section 6 we combine these results with some 3-manifold topology to compare the Thurston and Alexander norms, and complete the proof of Theorem 1.1. Examples are presented in Section 7.

Questions. Equality holds in Theorem 1.1 for fibered and alternating knots (see Section 7). Here are two questions for links $L$ with 2 or more components.

1. Do the Alexander and Thurston norms agree whenever $L$ is alternating?

2. Do the norms agree whenever $L$ is fibered? ${ }^{1}$

Notes and references. The Alexander polynomial of a knot was introduced in 1928 [2]. Fox treated the case of links and general groups via the free differential calculus [9]. For more on the Alexander polynomial of a knot, see [25,6,13] and [29]; for links, see [16] and [4]; and for 3 -manifolds, see [32]. References for fibered links include [27,30,15] and [12].

David Fried observed in the 1980s that the Thurston norm is related to the exponents of the Alexander polynomial in many examples. The Alexander ideal of a link is given in [9, II, 208-209]; see also [4, Prop. 9.16]. The first equality in (1.3) also appears in [32, §4.1], where it is proved by different methods (using Reidemeister torsion). Connections between the Alexander invariants and group cohomology, touched on in Section 3 below, are elucidated in [18].

The basic reference for the Thurston norm is [31]; see also [10,28]. Foliations provide a powerful geometric method for studying norm-minimizing surfaces; see [11]. Fibered faces of the Thurston norm ball are studied via a polynomial invariant in [22].

\footnotetext{
${ }^{1}$ N. Dunfield has announced a negative answer to this question [7].
} 
I would like to thank J. Christy for relating Fried's observation, and for useful conversations. Help with Table 1 in Appendix A was provided by D. Calegari, N. Dunfield and E. Hironaka.

Update. When this paper was first circulated (in 1998), D. Kotschick suggested that Theorem 1.1 could also be deduced (at least for closed, irreducible 3-manifolds) from the gauge theory results of of Kronheimer-Mrowka and Meng-Taubes [21,24]; the details of such a proof are presented by Vidussi in [33]. For more on interactions between the Alexander polynomial and Seiberg-Witten invariants, see [8,20] and [23].

\section{The Alexander invariants of a group}

Let $G$ be a finitely generated group, and let $\phi: G \rightarrow F$ be a surjective homomorphism to a free abelian group $F \cong \mathbb{Z}^{b}$. Let $\mathbb{Z}[F]$ be the integral group ring of $F$. In this section we recall the definitions of:

- the Alexander module $A_{\phi}(G)$ over $\mathbb{Z}[F]$,

- the Alexander ideal $I_{\phi}(G) \subset \mathbb{Z}[F]$, and

- the Alexander polynomial $\Delta_{\phi} \in \mathbb{Z}[F]$.

When $\phi: G \rightarrow \operatorname{ab}(G) \cong \mathbb{Z}^{b_{1}(G)}$ is the natural map to the maximal free abelian quotient of $G$, we denote these invariants simply by $A(G), I(G)$ and $\Delta_{G}$.

The Alexander module. Let $(X, p)$ be a pointed CW-complex with $\pi_{1}(X, p)=G$, let $\pi: \widetilde{X} \rightarrow X$ be the Galois covering space corresponding to $\phi: G \rightarrow F$, and let $\widetilde{p}=\pi^{-1}(p)$. The Alexander module is defined by

$$
A_{\phi}(G)=H_{1}(\tilde{X}, \widetilde{p} ; \mathbb{Z})
$$

equipped with the natural action of $F$ coming from deck transformations on $(\widetilde{X}, \widetilde{p})$.

Here is a more algebraic description of $A_{\phi}(G)$. For any subgroup $H \subset G$, let $m(H) \subset \mathbb{Z}[G]$ be the augmentation ideal generated by $\langle(h-1): h \in H\rangle$. Then we have

$$
A_{\phi}(G)=m(G) /(m(\operatorname{Ker} \phi) \cdot m(G)) \text {. }
$$

This quotient is manifestly a $G$-module, but it is also an $F$-module because $\mathbb{Z}[G] / m(\operatorname{Ker} \phi)=$ $\mathbb{Z}[F]$.

The correspondence between (2.1) and (2.2) is obtained by choosing a basepoint $* \in \widetilde{p}$, and identifying $(g-1) \in m(G)$ with the element of $H^{1}(\widetilde{X}, \widetilde{p})$ obtained by lifting the loop $g \in \pi_{1}(X)$ to a path in $\widetilde{X}$ running from $*$ to $g *$.

Now for any finitely-generated module $A$ over $\mathbb{Z}[F]$, one can choose a free resolution

$$
\mathbb{Z}[F]^{r} \stackrel{M}{\rightarrow} \mathbb{Z}[F]^{n} \rightarrow A
$$

the $i$ th elementary ideal $E_{i}(A) \subset \mathbb{Z}[F]$ is generated by the $(n-i) \times(n-i)$ minors of the matrix $M$. This ideal is independent of the resolution of $A$.

The Alexander ideal is the first elementary ideal of the Alexander module; that is,

$$
I_{\phi}(G)=E_{1}\left(A_{\phi}(G)\right) .
$$


The Alexander polynomial $\Delta_{\phi} \in \mathbb{Z}[F]$ is the greatest common divisor of the elements of the Alexander ideal. It is well-defined up to multiplication by a unit in $\mathbb{Z}[F]$. Equivalently, $\left(\Delta_{\phi}\right)$ is the smallest principal ideal containing $I_{\phi}(G) .^{2}$

\section{Characters and cohomology}

To give some intuition for the Alexander ideal, in this section we relate $I(G)$ to cohomology with twisted coefficients.

THEOREM 3.1.- A character $\rho \in \widehat{\mathrm{ab}(G)}$ lies in the variety $V(I(G))$ if and only if $\operatorname{dim} H^{1}\left(G, \mathbb{C}_{\rho}\right)>0$, or $\rho=1$ is trivial and $\operatorname{dim} H^{1}(G, \mathbb{C})>1$.

COROLLARY 3.2. - An Alexander polynomial in more than one variable defines the maximal hypersurface in the character variety such that $\operatorname{dim} H^{1}\left(G, \mathbb{C}_{\rho}\right)>0$ whenever $\Delta_{G}(\rho)=0$.

Twisted cohomology comes naturally from covering spaces. For example, let $M$ be a manifold and let $M_{A} \rightarrow M$ be a covering space with abelian Galois group $A$. Then $A$ acts on $H^{1}\left(M_{A}, \mathbb{C}\right)$, and we can try to decompose this action into irreducible pieces. The part of $H^{1}\left(M_{A}, \mathbb{C}\right)$ transforming by a nontrivial character $\rho \in \widehat{A}$ is isomorphic to $H^{1}\left(M, \mathbb{C}_{\rho}\right)$. By the result above, $H^{1}\left(M, \mathbb{C}_{\rho}\right)$ has positive dimension iff $\rho$ lies in $\widehat{A} \cap V(I(G))$.

Group cohomology. Given a $G$-module $B$, a crossed homomorphism $f: G \rightarrow B$ is a map satisfying $f\left(g g^{\prime}\right)=f(g)+g \cdot f\left(g^{\prime}\right)$. Such $f$ form the additive group $Z^{1}(G, B)$ of 1 -cocycles on $G$ with values in $B$. The coboundaries $B^{1}(G, B)$ are those $f$ given by $f(g)=g \cdot b-b$ for some $b \in B$; and the first cohomology group of $G$ is $H^{1}(G, B)=Z^{1}(G, B) / B^{1}(G, B)$.

The Alexander module satisfies

$$
\operatorname{Hom}_{G}\left(A_{\phi}(G), B\right) \cong Z^{1}(G, B)
$$

for any $F$-module $B$, considered as a $G$-module via $\phi: G \rightarrow F$. The natural isomorphism sends $h: A_{\phi}(G) \rightarrow B$ to $f(g)=h(g-1)$. Note that

$$
f\left(g g^{\prime}\right)=h\left(g g^{\prime}-1\right)=h\left((g-1)+g\left(g^{\prime}-1\right)\right)=h(g-1)+g \cdot h\left(g^{\prime}-1\right)=f(g)+g \cdot f\left(g^{\prime}\right),
$$

so $f$ is indeed a cocycle. To apply (3.1), note that $\mathbb{C}[\mathrm{ab}(G)]=\mathbb{Z}[\mathrm{ab}(G)] \otimes \mathbb{C}$ is the coordinate ring of the character variety

$$
\widehat{\operatorname{ab}(G)}=\operatorname{Hom}\left(\operatorname{ab}(G), \mathbb{C}^{*}\right) \cong\left(\mathbb{C}^{*}\right)^{b_{1}(G)} .
$$

Any character $\rho: \operatorname{ab}(G) \rightarrow \mathbb{C}^{*}$ determines a multiplicative action of $G$ on $\mathbb{C}$, and thus a $G$-module $B=\mathbb{C}_{\rho}$. The group $H^{1}\left(G, \mathbb{C}_{\rho}\right)$ classifies affine actions of the form

$$
g(z)=\rho(g) z+f(g),
$$

modulo those with fixed-points. By (3.1) we have

$$
\operatorname{dim}_{\mathbb{C}} A_{\phi}(G) \otimes \mathbb{C}_{\rho}=\operatorname{dim} Z^{1}\left(G, \mathbb{C}_{\rho}\right)=\operatorname{dim} H^{1}\left(G, \mathbb{C}_{\rho}\right)+ \begin{cases}0 & \text { if } \rho=1 \\ 1 & \text { otherwise }\end{cases}
$$

(The last term accounts for $\operatorname{dim} B^{1}\left(G, \mathbb{C}_{\rho}\right)$.)

\footnotetext{
${ }^{2}$ The definition of the Alexander polynomial uses the fact that $F$ is a free abelian group to insure that $\mathbb{Z}[F]$ is a unique factorization domain. If $F$ were to have torsion, then $\mathbb{Z}[F]$ would have zero divisors, and the greatest common divisor of an ideal would not be well-defined.
} 
Proof of Theorem 3.1. - The zero locus of $I(G)=E_{1}(A(G))$ coincides with those characters $\rho$ for which all $(n-1) \times(n-1)$ minors of a presentation matrix for $A(G)$ evaluate to zero, which occurs exactly when $A(G) \otimes \mathbb{C}_{\rho}$ has dimension 2 or more. Thus the theorem follows from the equation above.

See [18] for a more detailed development of the Alexander theory and group cohomology, containing the theorem above as a special case.

\section{The Alexander norm}

Let $\Lambda=\mathbb{Z}\left[s^{ \pm 1}\right]$ denote the group ring of $\mathbb{Z}$. The degree of a Laurent polynomial $\Delta \in \Lambda$ is the difference between its highest and lowest exponents, or $+\infty$ if $\Delta=0$.

Let $G$ be a finitely-generated group. A class $\phi \in H^{1}(G, \mathbb{Z}) \cong \operatorname{Hom}(G, \mathbb{Z})$ is primitive if $\phi(G)=\mathbb{Z}$. The Alexander polynomial of a primitive class satisfies

$$
b_{1}(\operatorname{Ker} \phi)=\operatorname{deg} \Delta_{\phi}
$$

Indeed, we have

$$
H^{1}(\operatorname{Ker} \phi, \mathbb{Q}) \cong\left(\Lambda /\left(\Delta_{\phi}\right)\right) \otimes \mathbb{Q} \cong \mathbb{Q}\left[s^{ \pm 1}\right] /\left(\Delta_{\phi}\right) ;
$$

see [25, Assertion 4].

Writing $I(G)=\left\langle f_{1}, \ldots, f_{n}\right\rangle$, we have

$$
\Delta_{\phi}=\operatorname{gcd}\left(\phi\left(f_{1}\right), \ldots, \phi\left(f_{n}\right)\right)
$$

and thus knowledge of the generators of the Alexander ideal allows one to determine $b_{1}(\operatorname{Ker} \phi)$. For example, if $\Delta_{G}=0$ then $b_{1}(\operatorname{Ker} \phi)=\infty$ for all $\phi \neq 0$.

Here is a restatement of (4.1) in terms of covering spaces as in (Section 3). Let $G$ be the fundamental group of a manifold $M$. Then the map $\phi: G \rightarrow \mathbb{Z}$ determines a covering space $M_{\phi} \rightarrow M$, and $H^{1}\left(M, \mathbb{C}_{\rho}\right)$ contributes to $H^{1}\left(M_{\phi}, \mathbb{C}\right)$ whenever $\rho$ factors through $\phi$. Counting these contributions gives

$$
b_{1}\left(M_{\phi}\right)=|\phi(\widehat{\mathbb{Z}}) \cap V(I(G))|=\operatorname{deg} \Delta_{\phi} .
$$

Here the intersections with $\widehat{\mathbb{Z}} \cong \mathbb{C}^{*}$ are counted with multiplicity, interpreting $V(I(G))$ as the scheme $\operatorname{Spec} \mathbb{C}[G] / I(G)$.

In this section we show $b_{1}(\operatorname{Ker} \phi)$ can be expressed in terms of the Alexander norm when $I(G)$ has a simple form. Let

$$
\Delta_{G}=\sum a_{\alpha} t^{\alpha}
$$

be the Alexander polynomial of $G$ written multiplicatively. (If $\alpha=\left(\alpha_{1}, \ldots, \alpha_{b}\right)$ denotes a typical element of $\operatorname{ab}(G) \cong \mathbb{Z}^{b}$, then $\left.t^{\alpha}=\left(t_{1}^{\alpha_{1}}, \ldots, t_{b}^{\alpha_{b}}\right)\right)$. The Alexander norm on $H^{1}(G, \mathbb{Z})$ is given by

$$
\|\phi\|_{A}=\sup \phi(\alpha-\beta),
$$

with the supremum over $(\alpha, \beta)$ such that $a_{\alpha} a_{\beta} \neq 0$. 
THEOREM 4.1. - Suppose $I(G)=m^{p}\left(\Delta_{G}\right) \neq(0)$, where $m=m(\mathrm{ab}(G))$ is the augmentation ideal. Then

$$
b_{1}(\operatorname{Ker} \phi)=\|\phi\|_{A}+p
$$

for all primitive $\phi$ inside the cone on the open faces of the Alexander norm ball.

(If the Alexander norm is identically zero, then equality holds for all $\phi$.)

Proof. - The map $\phi: G \rightarrow \mathbb{Z}$ extends to a map of group rings, $\phi: \mathbb{Z}[G] \rightarrow \Lambda$, and we have

$$
\phi\left(\Delta_{G}\right)=\Delta_{G}\left(s^{\phi}\right)=\sum a_{\alpha} s^{\phi(\alpha)} .
$$

The exponents of $\Delta_{G}\left(s^{\phi}\right)$ lie in the image of the Newton polytope of $\Delta_{G}$ under $\phi$, which is an interval of length $\|\phi\|_{A}$. Thus

$$
\operatorname{deg} \Delta_{G}\left(s^{\phi}\right)=\|\phi\|_{A}
$$

so long as the highest and lowest values of $\phi(\alpha)$ occur only once in (4.2). For $\phi$ in the cone on an open face of the norm ball, this uniqueness is automatic; indeed the extreme values $\phi(\alpha)$ and $\phi(\beta)$ are realized exactly when $\alpha-\beta$ is dual to the supporting hyperplane of the face.

To complete the proof, note that $\phi(m(\mathrm{ab}(G))=((s-1))$, so

$$
\left(\Delta_{\phi}\right)=I_{\phi}(G)=\phi(I(G))=\left((s-1)^{p} \Delta_{G}\left(s^{\phi}\right)\right)
$$

and therefore

$$
b_{1}(\operatorname{Ker} \phi)=\operatorname{deg} \Delta_{\phi}=p+\operatorname{deg} \Delta_{G}\left(s^{\phi}\right)=p+\|\phi\|_{A} .
$$

Failure of convexity. We will see in the next section that the Alexander ideal of a 3-manifold has the form stated in the theorem above. Thus for $G=\pi_{1}\left(M^{3}\right)$, the function $b_{1}(\operatorname{Ker} \phi)$ extends from primitive classes to a convex function on $H^{1}(G, \mathbb{R})$.

This convexity does not hold for general groups. For example, let $D_{\infty}$ be the semidirect product $\mathbb{Z} \ltimes \mathbb{Z}=\left\langle a, b: a b a^{-1}=b^{-1}\right\rangle$ (with $b_{1}\left(D_{\infty}\right)=1$ ), let $G=D_{\infty} \times D_{\infty}$, and let $(x, y)$ be multiplicative generators for $\operatorname{ab}(G)$. The Alexander ideal of $G$ is given by

$$
I(G)=\left\langle x^{2}-1, y^{2}-1,(x-1)(y-1)\right\rangle,
$$

so for primitive $\phi=(i, j) \in H^{1}(G, \mathbb{Z})$ we have

$$
\begin{aligned}
b_{1}(\operatorname{Ker} \phi) & =\operatorname{deg} \Delta_{\phi}(s)=\operatorname{deg}\left(\operatorname{gcd}\left(s^{2 i}-1, s^{2 j}-1,\left(s^{i}-1\right)\left(s^{j}-1\right)\right)\right) \\
& = \begin{cases}\operatorname{deg}(s-1)=1 & \text { if } i j \text { is odd }, \\
\operatorname{deg}\left(s^{2}-1\right)=2 & \text { otherwise. }\end{cases}
\end{aligned}
$$

This Betti number does not extend to a convex function on $\mathbb{R}^{2}$, since a bounded convex function is constant.

Question. How does $b_{1}(\operatorname{Ker} \phi)$ behave for a general group $G$ ? For example, does it exhibit a combination of convex and periodic behavior?

This question is suggested by the polynomial periodicity of $b_{1}$ for finite abelian coverings; cf. [17] and references therein. 


\section{The Alexander ideal of a 3-manifold}

THEOREM 5.1.- Let $G=\pi_{1}(M)$ be the fundamental group of a compact, orientable 3-manifold whose boundary is a union of tori. Then $I(G)=m^{p} \cdot\left(\Delta_{G}\right)$, where

$$
p= \begin{cases}0 & \text { if } b_{1}(M) \leqslant 1 \\ 1+b_{3}(M) & \text { otherwise }\end{cases}
$$

and $m=m(\mathrm{ab}(G))$ is the augmentation ideal.

Proof. - The case $M$ closed, $b_{1}(M) \geqslant 2$. We begin with the most interesting case.

The Alexander module $A(G)$ is naturally isomorphic to $H_{1}(M, p ; \mathbb{Z}[\mathrm{ab}(G)])$, where the coefficients are twisted by the multiplicative action of $\pi_{1}(M)$ on the group ring. To give a presentation for $A(G)$, choose a triangulation $\tau$ of $M$, and let $T$ be a maximal tree in the 1 -skeleton of $\tau$. Let $T^{\prime}$ be a maximal tree in the dual 1-skeleton - a tree whose vertices lie inside the tetrahedra of $\tau$, and whose edges join pairs of tetrahedra with common faces.

By collapsing $T$ to form a single 0 -cell $e_{0}$, and joining the 3 -simplices of $T^{\prime}$ to form a single 3 -cell $e_{3}$, we obtain a chain complex

$$
C_{3}^{1} \stackrel{\partial_{3}}{\rightarrow} C_{2}^{n} \stackrel{\partial_{2}}{\rightarrow} C_{1}^{n} \stackrel{\partial_{1}}{\rightarrow} C_{0}^{1}
$$

for $M$ over $\mathbb{Z}[\operatorname{ab}(G)]$. The upper indices give the numbers of cells; the numbers in dimensions 1 and 2 agree because, by our assumption on $\partial M$, we have $\chi(M)=0$. Then

$$
A(G)=H_{1}\left(M, e_{0}\right)=C_{1} / \partial_{1}\left(C_{2}\right),
$$

since all chains in $C_{1}$ are cycles rel $e_{0}$.

Choose bases for $C_{1}$ and $C_{2}$, and let $d_{i j}$ denote the determinant of the $(i, j)$-minor of the $n \times n$ matrix $\partial_{2}=D_{i j}$. Then the Alexander ideal is given simply by $I(G)=\left\langle d_{i j}\right\rangle$.

To show $I(G)=m(G)^{2}\left(\Delta_{G}\right)$, we will use the fact that $\partial_{1} \partial_{2}=\partial_{2} \partial_{3}=0$.

First note that for any 1 -cell $e_{1} \in C_{1}$, we have $\partial_{1}\left(e_{1}\right)=(1-g) e_{0}$, where $g \in \operatorname{ab}(G)$ is the 1 -cycle determined by $e_{1} \cup T$. Thus the boundary operator is given by the $1 \times n$ matrix

$$
\partial_{1}=\left(1-g_{1}, \ldots, 1-g_{n}\right)
$$

where $\left\langle g_{i}\right\rangle$ generate $\operatorname{ab}(G)$.

Next consider any 2-cell $e_{2} \in C_{2}$, let $e_{1}^{\prime}$ be its dual 1-cell in $T^{\prime}$, and let $h \in \operatorname{ab}(G)$ be the 1 -cycle determined by $e_{1}^{\prime} \cup T^{\prime}$. Since $e_{2}$ is the face of two tetrahedra in $\tau$, it occurs twice in $\partial e_{3}$, with total weight $(1-h)$. Thus $\partial_{3}$ can be expressed as an $n \times 1$ matrix

$$
\partial_{3}=\left(1-h_{1}, \ldots, 1-h_{n}\right),
$$

where again $\left\langle h_{i}\right\rangle$ generate $\operatorname{ab}(G)$.

By choosing new bases for the modules $C_{2}$ and $C_{1}$, we can assume that $h_{i}=g_{i}$ for all $i$, that $\left\langle g_{1}, \ldots, g_{b}\right\rangle$ gives a multiplicative basis for $\mathrm{ab}(G) \cong \mathbb{Z}^{b}$, and that $g_{i}=1$ for $i>b$.

Now fix a row $i$, and let $c_{j}$ be the $j$ th column of $D_{i j}$ with its $i$ th row omitted. Since $\partial_{3} \partial_{2}=0$, we have $\sum c_{j}\left(1-g_{j}\right)=0$. Applying elementary operations on columns, we find

$$
d_{i j}\left(1-g_{k}\right)=\operatorname{det}\left(c_{1}, \ldots, \widehat{c_{j}}, \ldots,\left(1-g_{k}\right) c_{k}, \ldots, c_{n}\right)
$$




$$
\begin{aligned}
& =\operatorname{det}\left(c_{1}, \ldots, \widehat{c_{j}}, \ldots,-\sum_{l \neq k}\left(1-g_{l}\right) c_{l}, \ldots, c_{n}\right) \\
& = \pm \operatorname{det}\left(c_{1}, \ldots,\left(1-g_{j}\right) c_{j}, \ldots, \widehat{c_{k}}, \ldots, c_{n}\right) \\
& = \pm d_{i k}\left(1-g_{j}\right) .
\end{aligned}
$$

From $\partial_{1} \partial_{2}=0$ we similarly obtain

$$
d_{i j}\left(1-g_{k}\right)= \pm d_{k j}\left(1-g_{i}\right) .
$$

Combining these calculations gives:

$$
d_{i j}\left(1-g_{k}\right)\left(1-g_{l}\right)= \pm d_{k l}\left(1-g_{i}\right)\left(1-g_{j}\right)
$$

for all indices $i, j, k$ and $l$. We will see (5.1) easily implies $I(G)=m(G)^{2}(\Delta)$.

First, for $k>b$ or $l>b$ we have $d_{11} \cdot 0=d_{k l}\left(1-g_{1}\right)^{2}$. Since $b_{1}(M)>0$, we know $g_{1} \neq 1$ and thus $d_{k l}=0$. So $I(G)$ is generated by $d_{i j}$ for $i, j \leqslant b$.

Second, from $d_{i i}\left(1-g_{j}\right)^{2}= \pm d_{j j}\left(1-g_{i}\right)^{2}$ we conclude the diagonal minors satisfy $d_{i i}= \pm\left(1-g_{i}\right)^{2} \Delta$, for some $\Delta$ independent of $i$. To make this conclusion, we need to be able to choose $i, j \leqslant b$ with $i \neq j$ (so that $1-g_{i}$ and $1-g_{j}$ are relatively prime), and it is here we use the assumption $b_{1}(M)=b \geqslant 2$.

Finally the equation

$$
d_{i j}\left(1-g_{1}\right)^{2}= \pm d_{11}\left(1-g_{i}\right)\left(1-g_{j}\right)= \pm\left(1-g_{1}\right)^{2}\left(1-g_{i}\right)\left(1-g_{j}\right) \Delta
$$

implies

$$
d_{i j}= \pm\left(1-g_{i}\right)\left(1-g_{j}\right) \Delta
$$

for all $i, j$. Since $\left\langle 1-g_{i}\right\rangle$ are generators for $m(\operatorname{ab}(G))$, we have shown that $I(G)=\left(d_{i j}\right)=$ $m(\operatorname{ab}(G))^{2} \cdot(\Delta)$ and $\Delta=\Delta_{G}$.

The case $\partial M \neq \emptyset, b_{1}(M) \geqslant 2$. In this case $\operatorname{dim} C_{3}=0, \operatorname{dim} C_{1}=n, \operatorname{dim} C_{2}=n-1$, and $D_{i j}$ is an $n \times(n-1)$ matrix. By deleting the $i$ th row and taking the determinant, $i=1, \ldots, n$, we obtain the generators $d_{i}$ of the Alexander ideal. From $\partial_{1} \partial_{2}=0$ we can still conclude that

$$
d_{i}\left(1-g_{j}\right)= \pm d_{j}\left(1-g_{i}\right)
$$

and therefore $I(G)=m(\operatorname{ab}(G)) \cdot\left(\Delta_{G}\right)$.

The case $b_{1}(M) \leqslant 1$. In this case $I(G)=\left(d_{1}\right)$ or $\left(d_{11}\right)$, so $I(G)$ is principal and therefore $I(G)=\left(\Delta_{G}\right)$.

\section{The Thurston norm}

In this section we complete the proofs of Theorem 1.1 comparing the Alexander and Thurston norms.

Proposition 6.1. - Let $\phi \in H^{1}(M, \mathbb{Z})$ be a primitive class with $b_{1}(\operatorname{Ker} \phi)$ finite. Then there exists a norm-minimizing surface $S \subset M$ with $[S]=\phi$ and with

$$
\begin{aligned}
& b_{0}(S)=1, \\
& b_{1}(S) \geqslant b_{1}(\operatorname{Ker} \phi), \quad \text { and } \\
& b_{2}(S)=b_{3}(M) .
\end{aligned}
$$


Proof. - Let $S$ be an oriented surface dual to $\phi$, with $\chi_{-}(S)=\|\phi\|_{T}$ and with $b_{0}(S)$ minimal among all such surfaces.

I. $S$ is connected. We begin by showing $b_{0}(S)=1$.

Write $S$ as a union of components $S=S_{1} \sqcup S_{2} \sqcup \cdots \sqcup S_{n}$, where $n=b_{0}(S)$. Let $C$ be the directed graph with a vertex $v_{i}$ for each component $M_{i}$ of $M-S$, and with an edge $e_{k}$ from $v_{i}$ to $v_{j}$ whenever $M_{i}$ and $M_{j}$ meet along a component $S_{k}$ of $S$. The edges are directed using the orientations of $M$ and $S$. There is a natural collapsing map

$$
\pi: M \rightarrow C \rightarrow S^{1}
$$

such that $\phi$ is the pullback of a generator of $H^{1}\left(S^{1}, \mathbb{Z}\right)$. (The map $C \rightarrow S^{1}$ sends each directed edge positively once around $S^{1}$.) Since $M$ is connected, so is $C$.

We claim $b_{1}(C)=1$. To see this, pull back to the universal cover of $S^{1}$, to obtain $\mathbb{Z}$-covering spaces

$$
M_{\phi} \rightarrow C_{\phi} \rightarrow \mathbb{R}
$$

The projection $M_{\phi} \rightarrow C_{\phi}$ admits a section, so we have $b_{1}\left(M_{\phi}\right) \geqslant b_{1}\left(C_{\phi}\right)$. But if $b_{1}(C)>1$, then $C_{\phi}$ has infinitely many loops and thus

$$
b_{1}(\operatorname{Ker} \phi)=b_{1}\left(M_{\phi}\right) \geqslant b_{1}\left(C_{\phi}\right)=\infty,
$$

contrary to our assumption that $b_{1}(\operatorname{Ker} \phi)$ is finite.

Next note that $C$ has no vertex of degree 1 . Indeed, the edge $e_{i}$ touching such a vertex would give a component of $S$ with $\left[S_{i}\right]=0$ in $H^{1}(M, \mathbb{Z})$; such superfluous components do not exist because $b_{0}(S)$ is minimal. Similarly, if two edges point towards the same vertex, then the corresponding surfaces satisfy $\left[S_{i}+S_{j}\right]=0$, again contradicting minimality of $b_{0}(S)$.

Therefore $C$ consists of a single $n$-cycle, and the collapsing map $C \rightarrow S^{1}$ has degree $n$. Since $\phi$ is primitive, we have $n=b_{0}(S)=1$.

II. $b_{1}(S) \geqslant b_{1}\left(M_{\phi}\right)$. The infinite cyclic covering space $M_{\phi} \rightarrow M$ can be constructed from compact submanifolds as

$$
M_{\phi}=\cdots N_{-1} \cup N_{0} \cup N_{1} \cup \cdots,
$$

where $\left\langle S_{i}=N_{i-1} \cap N_{i}\right\rangle$ are the lifts of $S$. Since $b_{1}\left(M_{\phi}\right)=b_{1}(\operatorname{Ker} \phi)$ is finite, the group $H_{1}\left(M_{\phi} ; \mathbb{Q}\right)$ is generated by the homology of some compact piece $N_{1} \cup N_{2} \cup \cdots \cup N_{k}$, as well as by $N_{-k} \cup \cdots \cup N_{-1}$. These two compact pieces are separated by $S$, so $H_{1}(S)$ must also generate $H_{1}\left(M_{\phi}\right)$. Therefore we have

$$
b_{1}(S) \geqslant b_{1}\left(M_{\phi}\right) .
$$

III. $b_{2}(S)=b_{3}(M)$. Since $\partial S$ rests on $\partial M$, we have $b_{2}(S)=b_{3}(M)$ when $S$ has a boundary. Now suppose $S$ is closed; we must show $M$ is closed.

If not, then $M$ has at least one torus boundary component, and this component lifts to each $N_{i}$. By Lefschetz duality, any orientable compact 3-manifold satisfies

$$
b_{1}(N) \geqslant \frac{1}{2} b_{1}(\partial N)
$$

(cf. [14, Ex. 28.15]), and therefore:

$$
b_{1}\left(N_{1} \cup N_{2} \cup \cdots \cup N_{k}\right) \geqslant k
$$


By Mayer-Vietoris, we have

$$
b_{1}\left(M_{\phi}\right) \geqslant b_{1}\left(N_{1} \cup N_{2} \cup \cdots \cup N_{k}\right)-2 b_{1}(S) \geqslant k-2 b_{1}(S) \rightarrow \infty
$$

as $k \rightarrow \infty$. But $b_{1}\left(M_{\phi}\right)$ is finite, a contradiction.

Proof of Theorem 1.1 (Comparison of norms). - Let $G=\pi_{1}(M)$. We may assume $\Delta_{G} \neq 0$ since otherwise the Alexander norm vanishes.

Theorem 5.1 states that $I(G)=m^{p(M)}\left(\Delta_{G}\right)$, so by Theorem 4.1 we have

$$
b_{1}(\operatorname{Ker} \phi)=\|\phi\|_{A}+p(M)
$$

for all primitive $\phi \in H^{1}(M, \mathbb{Z})$ outside a finite set of hyperplanes. Since the Alexander and Thurston norms are homogeneous and continuous, it suffices to prove the theorem for such $\phi$.

Let $S$ be the norm-minimizing surface dual to $\phi$ provided by Proposition 6.1; then we have

$$
b_{1}(\operatorname{Ker} \phi) \leqslant b_{1}(S)
$$

If $S$ is a 2-sphere or a 2-disk, then (6.2) and (6.1) imply $\|\phi\|_{A}=0$, so the theorem is automatic. Therefore we can assume $\chi(S) \leqslant 0$, which gives

$$
\begin{aligned}
\|\phi\|_{T} & =-\chi(S)=b_{1}(S)-b_{0}(S)-b_{2}(S) \geqslant b_{1}(\operatorname{Ker} \phi)-1-b_{3}(M) \\
& =\|\phi\|_{A}+p(M)-b_{3}(M)-1 .
\end{aligned}
$$

The inequality in the theorem then follows since $p(M)=b_{3}(M)+1$ for $b_{1}(M) \geqslant 2$, and $p(M)=0$ otherwise.

In the case of a fibration, $b_{1}(\operatorname{Ker} \phi)=b_{1}(S)$, so equality holds.

\section{Examples: manifolds, knots and links}

In this section we discuss examples of the Alexander polynomial and the Thurston norm. We use the shorthand $\Delta_{M}$ for $\Delta_{G}$ when $G=\pi_{1}(M)$, and $\Delta_{L}$ when $G=\pi_{1}\left(S^{3}-\mathcal{N}(L)\right)$ is the fundamental group of a link complement.

We begin with some simple closed 3-manifolds.

1. The 3-torus. For $M=S^{1} \times S^{1} \times S^{1}$, we have $\Delta_{M}=1$. (More generally $I(G)=m(G)^{n-1}$ when $G=\mathbb{Z}^{n}$.) The homology $H_{2}(M)$ is generated by tori, so the Thurston and Alexander norms both vanish identically.

2. Doubled handlebodies. Let $M$ be the connect sum of $n>1$ copies of $S^{2} \times S^{1}$. Then $G=\pi_{1}(M)$ is a free group on $n$ generators, and $\Delta_{M}=0$. Indeed, a crossedhomomorphism $f: G \rightarrow \mathbb{C}_{\rho}$ can be specified arbitrarily on the generators of $G$; thus $\operatorname{dim} Z^{1}\left(G, \mathbb{C}_{\rho}\right)=n>1$ for all $\rho$, so $\Delta_{G}$ must vanish identically by Theorem 3.1.

Clearly $\mathrm{H}_{2}(M)$ is generated by spheres, so the Thurston and Alexander norms both vanish.

3. Circle bundles. Let $G=\pi_{1}\left(S_{g}\right)$ be the fundamental group of a surface of genus $g \geqslant 2$. Since $G$ admits a presentation with $2 g$ generators and one relation, we have $\operatorname{dim} Z^{1}\left(G, \mathbb{C}_{\rho}\right) \geqslant 2 g-1>1$ for all $\rho$, and therefore $\Delta_{G}=0$ just as for a free group.

Now let $M \rightarrow S_{g}$ be a nontrivial circle bundle over $S_{g}$. The cohomology of $S_{g}$ pulls back to $M$, so $\Delta_{M}=0$ as well. The preimages of circles on $S_{g}$ generate $H_{2}(M)$, so the Thurston and Alexander norms both vanish. 
4. Solvemanifolds. Let $M \rightarrow S^{1}$ be a torus bundle over the circle. Then $G=\pi_{1}(M)=\mathbb{Z}^{2} \ltimes \mathbb{Z}$, where $\mathbb{Z}$ acts on $\mathbb{Z}^{2}$ by a matrix $A \in S L_{2}(\mathbb{Z})$. If $A$ is hyperbolic, then $M$ is a solvemanifold, $b_{1}(G)=1$ and $\Delta_{G}(t)=\operatorname{det}(t I-A)$ is the characteristic polynomial of $A$.

The torus fiber generates $H_{2}(M)$, so the Thurston norm vanishes identically. Thus $\|\phi\|_{A}=\|\phi\|_{T}+2$ on the generator of $H^{1}(M)$, so equality holds in Theorem 1.1.

5. Surface bundles. More generally, let $M \rightarrow S^{1}$ be a surface bundle over the circle, with fiber $S_{g}$ and $b_{1}(M)=1$. Then $\Delta_{G}(t)=\operatorname{det}(t I-A)$ is the characteristic polynomial for the monodromy acting on $H^{1}\left(S_{g}, \mathbb{Z}\right)$. On the generator of $H^{1}(M)$ we have

$$
\|\phi\|_{A}=2 g=\chi\left(S_{g}\right)+2=\|\phi\|_{T}+2,
$$

so again equality holds in Theorem 1.1.

6. Nilmanifolds. Let $M \rightarrow S^{1} \times S^{1}$ be the Heisenberg manifold with

$$
G=\pi_{1}(M)=\langle a, b, c:[a, b]=c,[a, c]=[b, c]=1\rangle .
$$

Then $b_{1}\left(M^{3}\right)=2, \Delta_{G}=1$ and $I(G)=m(G)^{2}$. The Thurston and Alexander norms both vanish identically.

In the preceding examples, the multiplicity of $I(G)$ at $\rho=1$ was $b_{1}(G)-1$; this rule of thumb fails for the Heisenberg group.

7. $S^{2} \times S^{1}$. Since $\Delta_{\mathbb{Z}}=1$, we have $\Delta_{M}=1$ for $M=D^{2} \times S^{1}$ and $M=S^{2} \times S^{1}$. Thus the Thurston and Alexander norms vanish identically for these manifolds. Strict inequality holds in Theorem 1.1, since $b_{1}(M)=1$.

Knots and links. Next we consider classical link complements. Let $L \subset S^{3}$ be a smoothly embedded link with $b$ components, let $M=S^{3}-\mathcal{N}(L)$ be the compact 3-manifold obtained by deleting a tubular of $L$, and let $G=\pi_{1}(M)$. Choose an ordering for the components of $L$ and an orientation for their meridians; then we have a multiplicative basis $\left\langle t_{1}, \ldots, t_{b}\right\rangle$ for $H_{1}(M, \mathbb{Z})=\mathrm{ab}(G)$, and hence a natural isomorphism between the group ring $\mathbb{Z}[\operatorname{ab}(G)]$ and the ring of Laurent polynomials $\mathbb{Z}\left[t_{1}^{ \pm 1}, \ldots, t_{b}^{ \pm 1}\right]$. The Alexander polynomial of the link is customarily written in terms of this basis, as

$$
\Delta_{L}(t)=\sum a_{\alpha} t^{\alpha}
$$

where the sum extends over all multi-indices $\alpha=\left(\alpha_{1}, \ldots, \alpha_{b}\right) \in \mathbb{Z}^{b}$.

Let $\phi=\left(\phi_{1}, \ldots, \phi_{b}\right) \in H^{1}(M, \mathbb{Z})$, in coordinates where $\phi(\alpha)=\sum \phi_{i} \alpha_{i}$. Then $\|\phi\|_{T}$ is the minimum of $\chi_{-}(S)$ over all $S \subset M$ whose boundary runs $\phi_{i}$-times around $L_{i}$.

Let $N\left(\Delta_{G}\right) \subset H_{1}(M, \mathbb{R}) \cong \mathbb{R}^{b}$ be the Newton polytope of the Alexander polynomial, i.e. the smallest convex set containing $\alpha$ whenever $a_{\alpha} \neq 0$. Then $\phi\left(N\left(\Delta_{G}\right)\right)$ is an interval on the real axis, and its length gives the Alexander norm:

$$
\|\phi\|_{A}=\operatorname{length}\left(\phi\left(N\left(\Delta_{G}\right)\right)\right) .
$$

Since the Alexander polynomial of a 3-manifold is symmetric [3], [32, p. 323], the Alexander norm ball is dual to the Newton polytope (up to a scale factor of 2).

Here are some basic principles that can sometimes be used to show $2 g(K)=\operatorname{deg} \Delta_{K}$ for a knot, and that the Alexander and Thurston norms agree for a link with 2 or more components.

(a) For a link, it suffices to exhibit a dual surface with

$$
\chi_{-}\left(S_{\phi}\right)=\|\phi\|_{A}
$$


for each $\phi$ in the finite set of extreme points (or vertices) of the Alexander norm ball. If this can be done, then global equality between the norms follows by convexity and Theorem 1.1.

In the case where $\|\cdot\|_{A}$ is degenerate, one must first check equality on a basis for the subspace $V$ where the Alexander norm vanishes, and then on the vertices of the image of the norm ball in $H^{1}(M, \mathbb{Z}) / V$.

(b) For any class $\phi$ with $\left|\phi_{i}\right| \leqslant 1$ for all $i$, a candidate surface $S_{\phi}$ can be constructed using Seifert's algorithm (see, e.g. [29, Ch. 5]).

(c) If links $L$ and $L^{\prime}$ have homeomorphic complements, then it suffices to check equality of norms for either one. (This principle is often useful when a non-alternating link is equivalent to an alternating one.)

(d) Crowell and Murasugi have shown for alternating links we have $\|\phi\|_{T}=\|\phi\|_{A}$ whenever $\left|\phi_{i}\right|=1$ for all $i$. They have also shown $2 g(K)=\operatorname{deg} \Delta_{K}$ for alternating knots [5,26]. In both cases an optimal surface is obtained using Seifert's algorithm, so this check can also be carried out using (b).

Tables of knots and links. We now turn to examples drawn from the tables in [29]. These tables give diagrams for the prime knots up to 10 crossings and links up to 9 crossings, together with their Alexander polynomials. Notation such as 96 indicates the 6 th link with 9 crossings and 3 components.

It is known that $2 g(K)=\operatorname{deg} \Delta_{K}$ for all knots with 10 crossings or less (see e.g. [11]), so all knots in the tables give examples where the inequality of Theorem 1.1 is equality.

Using principles (a)-(d) above, one can systematically check that the Thurston and Alexander norms agree for 128 of the 131 links on 2 or more components in [29]. To facilitate the application of (c), Appendix A lists links with homeomorphic complements; it also corrects two of the Alexander polynomials given in Rolfsen.

The few links that require a finesse beyond the straightforward application of (a)-(d) are included in the examples below. In summary we find:

THEOREM 7.1. - The Thurston and Alexander norms agree for all the tabulated links with 9 or fewer crossings except $9_{21}^{3}$, and possibly $9_{41}^{2}, 9_{50}^{2}$, and $9_{15}^{3}$.

For the last three links, the question of equality of norms is not resolved by principles (a)-(d); these links have extreme classes with $\left|\phi_{i}\right|>1$ for some $i$, so Seifert's algorithm does not apply.

The Thurston and Alexander norms disagree for $9_{21}^{3}$ because its Alexander polynomial is trivial, and many more knots and links with trivial Alexander polynomial can be easily constructed; see examples 9 and 10 below.

\section{Examples.}

1. The link $9_{42}^{2}$. Let $L=L_{1} \cup L_{2}$ be the alternating link shown in Fig. 1. Its Alexander polynomial is $\Delta_{L}=\sum a_{i j} t_{1}^{i} t_{2}^{j}$ where

$$
a_{i j}=\left[\begin{array}{ccccc}
0 & -1 & 3 & -3 & 1 \\
-1 & 4 & -7 & 4 & -1 \\
1 & -3 & 3 & -1 & 0
\end{array}\right]
$$

The Newton polytope $N\left(\Delta_{L}\right)$ can be visualized as the convex hull of the nonzero entries above; it has faces of slope $\infty, 0$ and 1 . The extreme points of the Alexander norm ball are therefore proportional to $\phi=(1,0),(0,1)$ and $(-1,1)$. The Alexander norms of these extreme classes are given by $\|\phi\|_{A}=4,2$ and 4 respectively. 

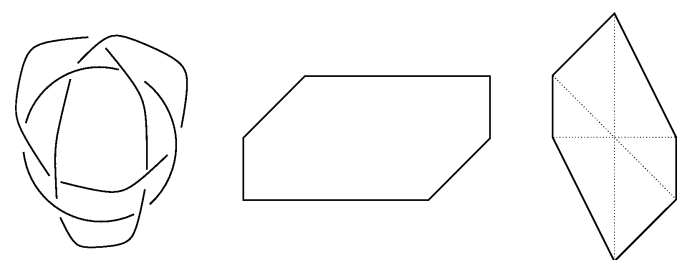

Fig. 1. The link $9_{42}^{2}$, its Newton polygon and its norm ball.
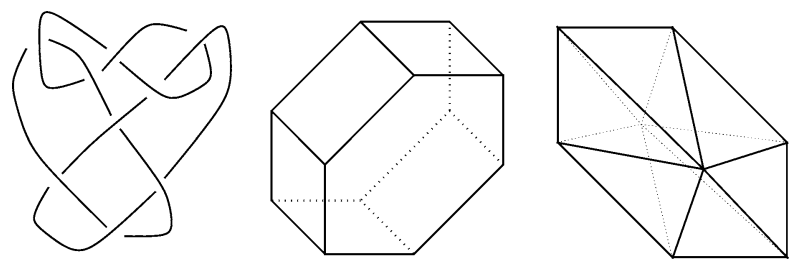

Fig. 2. The link $9_{6}^{3}$, its Newton polytope and its norm ball.

We now verify that the Thurston and Alexander norms agree for this link. It suffices to produce, for each of these 3 extreme classes $\phi$, a surface with $\left[S_{\phi}\right]=\phi$ satisfying $\chi_{-}\left(S_{\phi}\right)=\|\phi\|_{A}$.

For the class $\phi=(1,0)$, span the trefoil component $L_{1}$ of $L$ by its standard Seifert surface $T$ (with 2 regions, one of them unbounded). Then $T$ is a torus with one boundary component, pierced 3 times by $L_{2}$; removing these intersections we obtain a torus with 4 holes $S_{\phi}$ such that

$$
\chi_{-}\left(S_{\phi}\right)=4=\|\phi\|_{A} .
$$

Similarly, a standard disk spanning the unknotted component $L_{2}$ is pierced 3 times by $L_{1}$, producing a surface for $\phi=(0,1)$ with $\chi_{-}\left(S_{\phi}\right)=2=\|\phi\|_{A}$, so the norms agree on this class as well. Finally, since the link is alternating, the equality $\|\phi\|_{A}=\|\phi\|_{T}=4$ is automatic for $\phi=(-1,1)$ by the result of Crowell-Murasugi (principle (d) above).

Having checked equality at the extreme points of the Alexander norm ball, we conclude that the Thurston and Alexander norms coincide for this link.

2. The link $9_{6}^{3}$. This 3 -component link has Alexander polynomial $\Delta_{L}=\sum a_{i j k} t_{1}^{i} t_{2}^{j} t_{3}^{k}$, where

$$
a_{i j 0}=\left[\begin{array}{ccc}
0 & 1 & -1 \\
1 & -3 & 2 \\
-1 & 2 & 0
\end{array}\right], \quad a_{i j 1}=\left[\begin{array}{ccc}
0 & -2 & 1 \\
-2 & 3 & -1 \\
1 & -1 & 0
\end{array}\right]
$$

See Fig. 2. The top component of the link corresponds to the distinguished direction in $H^{1}(M, \mathbb{Z})$. The extreme points of the norm ball are proportional to $\phi=(1,0,0)$, $(0,1,0),(0,0,1)$ and $(0,-1,1)$, with $\|\phi\|_{A}=1,2,2$ and 2 respectively.

To check equality of the Thurston and Alexander norms for this link, it suffices to exhibit surfaces satisfying $\chi_{-}\left(S_{\phi}\right)=\|\phi\|_{A}$ for the 4 extreme classes above. For the first three classes, we note that each component of $L$ is spanned by a disk, pierced 2 or 3 times by the rest of the link, yielding a surface with $\chi_{-}\left(S_{\phi}\right)=1$ or 2 , in agreement with the Alexander norm. For the final class $\phi=(0,-1,1)$, take an annulus spanning the right and left components of $L$; it is pierced twice by the top component, yielding a surface with $\chi_{-}\left(S_{\phi}\right)=2=\|\phi\|_{A}$ in this case as well. 

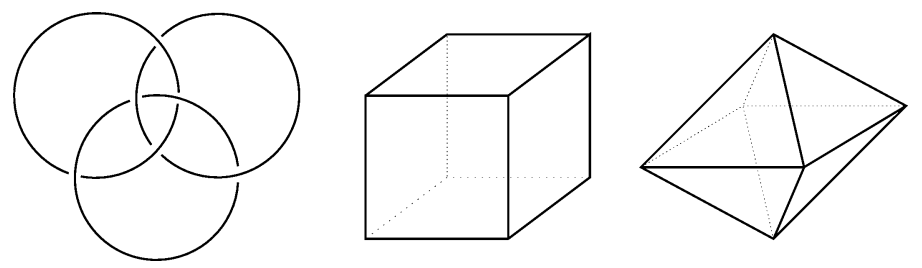

Fig. 3. The Borromean rings, their Newton polytope and its norm ball.
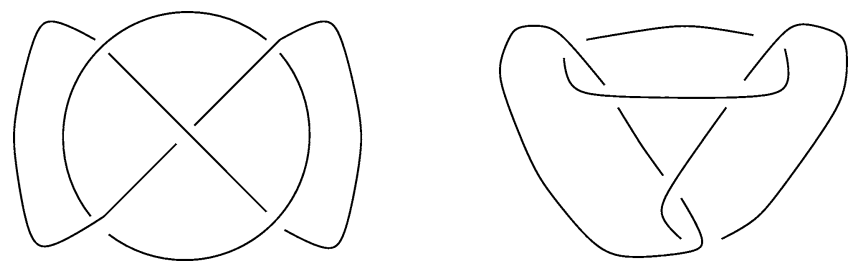

Fig. 4. The links $5_{1}^{2}$ and $6_{3}^{3}$.

Note: even though the link is alternating, the result of Crowell-Murasugi was not used, because there was no extreme class with $\left|\phi_{i}\right|=1$ for all $i$.

3. The Borromean rings. The Alexander polynomial for the Borromean rings is

$$
\Delta_{L}(t)=\left(t_{1}-1\right)\left(t_{2}-1\right)\left(t_{3}-1\right),
$$

so its Newton polytope is the cube $[0,1]^{3}$. The unit ball of the Alexander norm is therefore an octahedron (Fig. 3), and

$$
\left\|\left(\phi_{1}, \phi_{2}, \phi_{3}\right)\right\|_{A}=\left|\phi_{1}\right|+\left|\phi_{2}\right|+\left|\phi_{3}\right| .
$$

One can use fibrations to check equality of the Thurston and Alexander norms for this link. Indeed, $M=S^{3}-\mathcal{N}(L)$ is homeomorphic to $T^{3}-\mathcal{N}\left(L^{\prime}\right)$, where $T^{3}=\mathbb{R}^{3} / \mathbb{Z}^{3}$ is the 3 -torus and $L^{\prime}$ consists of three disjoint closed geodesics parallel to the coordinate axes. Any nonzero cohomology class in $H^{1}\left(T^{3}, \mathbb{Z}\right)$ is represented by a fibration $T^{3} \rightarrow S^{1}$, which restricts to a fibration $M \rightarrow S^{1}$ so long as the fibers are transverse to $\partial M$. Thus $\|\phi\|_{T}=\|\phi\|_{A}$ for all $\phi \in H^{1}(M, \mathbb{Z})$ outside the planes $\left(\phi_{i}=0\right)$; by continuity, the two norms coincide everywhere. Compare [4, p. 132], [31, p. 111].

4. The link $5_{1}^{2}$. For this link we have

$$
\Delta_{L}\left(t_{1}, t_{2}\right)=\left(1-t_{1}\right)\left(1-t_{2}\right)
$$

Since $\Delta_{L}$ vanishes identically along the line $t_{2}=1$, we have $\Delta_{\phi}(s)=0$ for $\phi=(1,0)$. Nevertheless, $\|\phi\|_{A}=1$, and indeed the Thurston and Alexander norms for $L$ agree.

This example shows the genus of a surface is controlled more precisely by the Alexander norm than by the 1-variable Alexander polynomial of a cohomology class.

5. The link $6_{3}^{3}$. The complement of this link is $M=S^{1} \times F$, where $F$ is a sphere with 3 holes. Thus its Thurston and Alexander norms agree.

6. The link $9_{52}^{2}$. The extreme class $\phi=(1,-1)$ for this link has Alexander norm 2, but Seifert's algorithm gives a surface of Thurston norm 4 when applied to the projection in [29]. To obtain an optimal surface, redraw the projection as shown in Fig. 5, and orient 


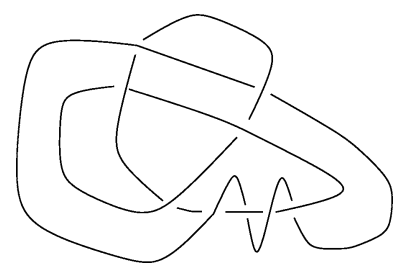

Fig. 5. The link $9_{52}^{2}$ is spanned by a surface of genus 1 .

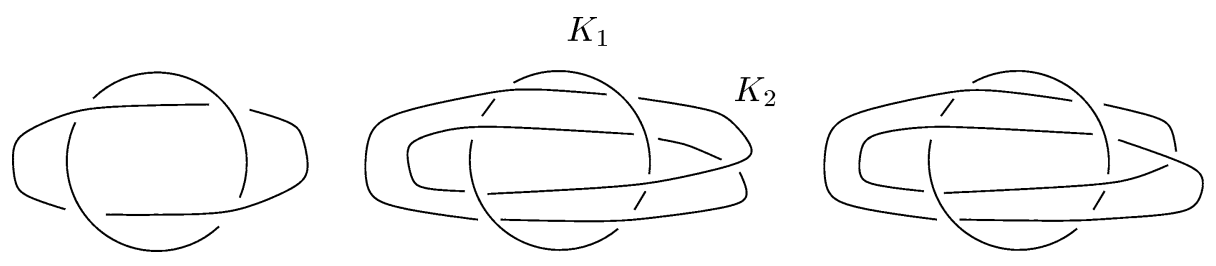

Fig. 6. The link $4_{1}^{2}$ and its satellites $9_{53}^{2}$ and $9_{61}^{2}$.

the components in opposite directions. The new diagram then has 7 Seifert circles, so it produces a Seifert surface with $\chi(S)=7-9=-2$ as desired.

7. The satellite links $9_{53}^{2}$ and $9_{61}^{2}$. To study the Thurston and Alexander norms for a satellite link, it is advantageous to cut the link complement into atoroidal pieces.

The link $L=9_{53}^{2}$ is a satellite of the torus link $4_{1}^{2}$ (Fig. 6). Its Alexander polynomial is

$$
\Delta_{L}=\left(1+t_{1} t_{2}^{2}\right)\left(1+t_{1}^{2} t_{2}\right) .
$$

The extreme points of the Alexander norm ball are represented by $\phi=(2,-1)$ and $(-1,2)$.

Let $\left(\ell_{i}, m_{i}\right)$ be the longitude and meridian of $K_{i}$. We will construct a surface $S$ dual to $\phi=(2,-1)$ with

$$
\partial S=\left(2 \ell_{1}+4 m_{1}\right)-\left(\ell_{2}+8 m_{2}\right)
$$

and with $\chi_{-}(S)=\|\phi\|_{A}=3$.

Let $T \subset M=S^{3}-\mathcal{N}(L)$ be the incompressible torus separating $L$ into an outer circle $K_{1}$ and an inner doubled loop $K_{2}$. Let $(\ell, m)$ be a framing for $T$ such that $m$ bounds a disk inside and $\ell$ bounds a disk outside.

Because $4_{1}^{2}$ is the $(2,4)$-torus link, there is an annulus $A$ with between $K_{1}$ and $T$ with

$$
\partial A=\left(\ell_{1}+2 m_{1}\right)-(\ell+2 m) .
$$

Similarly, there is an annulus $B$ between $T$ and $K_{2}$ with

$$
\partial B=(2 \ell+m)-\left(\ell_{2}+2 m_{2}\right) .
$$

Finally, there is a pair of pants $P$ between $T$ and $K_{2}$ with

$$
\partial P=m-2 m_{2} .
$$

Combining these surfaces, we obtain a 2-chain with

$$
\partial(2 A \cup B \cup 3 P)=\left(2 \ell_{1}+4 m_{1}\right)-\left(\ell_{2}+8 m_{2}\right) .
$$




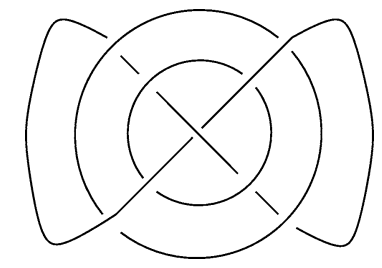

Fig. 7. The Alexander and Thurston norms differ for $9_{21}^{3}$.

Table 1

\begin{tabular}{|c|c|c|c|c|c|c|c|c|c|c|c|c|c|}
\hline \multicolumn{14}{|c|}{ Links in the same column have homeomorphic complements } \\
\hline $4_{1}^{2}$ & $5_{1}^{2}$ & $6_{1}^{2}$ & $6_{3}^{2}$ & $7_{3}^{2}$ & $7_{4}^{2}$ & $7_{5}^{2}$ & $6_{1}^{3}$ & $6_{2}^{3}$ & $6_{3}^{3}$ & $7_{1}^{3}$ & $8_{9}^{3}$ & $9_{55}^{2}$ & $9_{59}^{2}$ \\
\hline $7_{7}^{2}$ & $7_{8}^{2}$ & $9_{49}^{2}$ & $8_{16}^{2}$ & $9_{46}^{2}$ & $9_{44}^{2}$ & $9_{48}^{2}$ & $8_{8}^{3}$ & $9_{18}^{3}$ & $8_{7}^{3}$ & $9_{14}^{3}$ & $9_{19}^{3}$ & $9_{56}^{2}$ & $9_{60}^{2}$ \\
\hline $9_{43}^{2}$ & $8_{15}^{2}$ & & $9_{45}^{2}$ & & & & $9_{13}^{3}$ & & & & & & \\
\hline & $9_{47}^{2}$ & & & & & & $9_{17}^{3}$ & & & & & & \\
\hline
\end{tabular}

Cut-and-paste yields the desired embedded surface $S$, with $\chi(S)=2 \chi(A)+\chi(B)+$ $\chi(3 P)=-3$.

By symmetry, $\|\phi\|_{A}=\|\phi\|_{T}$ for the other extreme class $\phi=(-1,2)$. Therefore the Thurston and Alexander norms agree for $9_{53}^{3}$.

A similar argument shows the norms also agree for $9_{61}^{2}$.

8. The link $9_{51}^{2}$. Like the three exceptions in Theorem 7.1, the link $9_{51}^{2}$ has an extreme class with $\left|\phi_{i}\right|>1$ for some $i$, so Seifert's algorithm does not apply. However we have recently shown that the Thurston and Alexander norms coincide for $9_{51}^{2}$, using the fact that this link is fibered [22, §11].

9. A link whose norms disagree. The Thurston and Alexander norms differ for $L=9_{21}^{3}$ (Fig. 7). Indeed, $\Delta_{L}=0$, so the Alexander norm of $L$ is trivial; but $L$ contains $5_{1}^{2}$ as a sublink, so its Thurston norm is nontrivial. (In fact $L$ is a satellite of $5_{1}^{2}$.)

This example also shows the Alexander norm can increase under passage to a sublink.

10. Trivial Alexander polynomials. Starting with 11 crossings there are many knots with $\Delta_{K}=1$ (see e.g. [29, p. 167]), and these provide examples of the strict inequality $\operatorname{deg} \Delta_{K}(t)<2 g(K)$. By clasping together two such knots, one can obtain many links with trivial Alexander polynomial and $\|\phi\|_{A}<\|\phi\|_{T}$.

\section{Appendix A. Links with homeomorphic complements}

A link complement $M=S^{3}-\mathcal{N}(L)$ can sometimes be embedded in $S^{3}$ in more than one way. For an intrinsic study of 3-manifolds, it is helpful to know which complements appear more than once in Rolfsen's tables. These coincidences are summarized in Table 1.

In the first row each homeomorphism type is represented by a link with the minimum number of crossings. Any link below the first row can be modified by surgery to obtain an equivalent link above it, usually with fewer crossings. To indicate these simplifications, we use the link projections shown in [29]. In each projection we label the top-most component $A$, then the next component $B$, and so on to the bottom. The components along which we will perform surgery are unknotted and their projections are simple; we orient them in the counter-clockwise sense. 
A surgery instruction such as $B$ - means: cut open $S^{3}$ along a disk spanning $B$, twist once in the negative direction (using the orientation of $B$ ), then reglue to obtain a new link $L^{\prime}$ with the same complement as $L$.

The links below the first row of the table can then be classified as follows:

- Simplified by $A+: 7_{7}^{2}, 7_{8}^{2}, 8_{15}^{2}, 8_{16}^{2}, 9_{43}^{2}, 9_{44}^{2}, 9_{45}^{2}, 9_{46}^{2}, 9_{47}^{2}, 9_{56}^{2}, 9_{60}^{2}$.

- Simplified by $A-: 9_{48}^{2}, 9_{49}^{2}, 8_{7}^{3}, 8_{8}^{3}, 9_{13}^{3}, 9_{14}^{3}, 9_{18}^{3}, 9_{19}^{3}$.

- The link $9_{17}^{3}$ : after $A+, B+, A+$, this link becomes $6_{1}^{3}$.

Almost all the other links in Rolfsen's tables can be distinguished using their Alexander polynomials, their hyperbolic volumes, and the shapes of their cusps. The Alexander polynomials are tabulated in Rolfsen; the hyperbolic data is tabulated in [1]. (As pointed out to us by N. Dunfield, there are some misprints in Rolfsen's tables; the Alexander polynomial for $9_{55}^{2}$ should actually be the same as that for $9_{56}^{2}$, and the matrix

$$
\left[\begin{array}{cccccc}
0 & 0 & 1 & -1 & 0 & 1 \\
1 & 0 & -1 & 1 & 0 & 0
\end{array}\right]
$$

gives the Alexander polynomial for $9_{59}^{2}$.)

There is one pair of links $\left(L_{1}, L_{2}\right)$ whose complements are not distinguished by these invariants, namely $\left(9_{53}^{2}, 9_{61}^{2}\right)$ (Fig. 6). These two links are satellites of $4_{1}^{2}$. Indeed, for $i=1,2$ the manifold $M_{i}=S^{3}-\mathcal{N}\left(L_{i}\right)$ splits along a torus $T_{i}$ into two copies of $N=S^{3}-\mathcal{N}\left(4_{1}^{2}\right)$.

To distinguish these manifolds, first note that $N$ is canonically Seifert fibered. Thus $\partial N$ has a natural foliation by simple closed curves, each generating the central subgroup $\mathbb{Z} \subset \pi_{1}(N)$. Since the two pieces of $M_{i}-\mathcal{N}\left(T_{i}\right)$ are both homeomorphic to $N$, the torus $T_{i}$ carries two natural foliations. Let $n\left(M_{i}\right)$ denote the intersection number of a leaf in one foliation with a leaf in the other. By uniqueness of the torus decomposition [19, Ch. IX], $n\left(M_{i}\right)$ is an invariant of $M_{i}$ (up to sign). One can check that $n\left(M_{1}\right)=3$ while $n\left(M_{2}\right)=5$, so in fact the links $9_{53}^{2}$ and $9_{61}^{2}$ have different complements.

\section{REFERENCES}

[1] Adams C., Hildebrand M., Weeks J., Hyperbolic invariants of knots and links, Trans. Amer. Math. Soc. 326 (1991) 1-56.

[2] AleXAnder J.W., Topological invariants of knots and links, Trans. Amer. Math. Soc. 30 (1928) 275306.

[3] BLANCHFIELD R.C., Intersection theory of manifolds with operators with applications to knot theory, Ann. of Math. 65 (1957) 340-356.

[4] Burde G., Zieschang H., Knots, Walter de Gruyter, 1985.

[5] Crowell R.H., Genus of alternating link types, Ann. of Math. 69 (1959) 258-275.

[6] Crowell R.H., Fox R.H., Introduction to Knot Theory, Springer-Verlag, 1977.

[7] Dunfield N., Alexander and Thurston norms of fibered 3-manifolds, Pacific J. Math., To appear.

[8] Fintushel R., Stern R., Knots, links and 4-manifolds, Invent. Math. 134 (1998) 363-400.

[9] Fox R.H., Free differential calculus I, II, III, Ann. of Math. 57, 59, 64 (1953, 1954, 1956) 547-560, 196-210, 407-419.

[10] FRIED D., Fibrations over $S^{1}$ with pseudo-Anosov monodromy, in: Travaux de Thurston sur les surfaces, Astérisque, Vol. 66-67, 1979, pp. 251-265.

[11] Gabai D., Foliations and genera of links, Topology 23 (1984) 381-394.

[12] Gabai D., Detecting fibred links in $S^{3}$, Comment. Math. Helv. 61 (1986) 519-555.

[13] Gordon C., Some aspects of classical knot theory, in: Knot Theory (Proc. Sem., Plans-sur-Bex, 1977), Lecture Notes in Math., Vol. 685, Springer-Verlag, 1978.

[14] Greenberg M.J., Harper J.R., Algebraic Topology, Benjamin/Cummings Publishing, 1981.

$4^{\text {e }}$ SÉRIE - TOME $35-2002-\mathrm{N}^{\circ} 2$ 
[15] HARER J.L., How to construct all fibered knots and links, Topology 21 (1972) 263-280.

[16] Hillman J.A., Alexander Ideals of Links, Lecture Notes in Math., Vol. 895, Springer-Verlag, 1981.

[17] HiRONAKa E., Torsion points on an algebraic subset of an affine torus, Internat. Math. Res. Notices (1996) 953-982.

[18] HironaKa E., Alexander stratifications of character varieties, Ann. Inst. Fourier (Grenoble) 47 (1997) $555-583$.

[19] JACO W., Lectures on 3-Manifold Topology, American Mathematical Society, 1980.

[20] Kronheimer P., Embedded surfaces and gauge theory in three and four dimensions, in: Surveys in Differential Geometry, Vol. III (Cambridge, MA, 1996), Int. Press, 1998, pp. 243-298.

[21] Kronheimer P., Mrowka T., Scalar curvature and the Thurston norm, Math. Res. Lett. 4 (1997) 931-937.

[22] McMullen C., Polynomial invariants for fibered 3-manifolds and Teichmüller geodesics for foliations, Ann. Scient. Éc. Norm. Sup. 33 (2000) 519-560.

[23] McMullen C., Taubes C., 4-manifolds with inequivalent symplectic forms and 3-manifolds with inequivalent fibrations, Math. Res. Lett. 6 (1999) 681-696.

[24] Meng G., Taubes C.H., $\underline{S W}=$ Milnor torsion, Math. Res. Lett. 3 (1996) 661-674.

[25] Milnor J., Infinite cyclic coverings, in: Collected Papers, Vol. 2. The Fundamental Group, Publish or Perish, 1994, pp. 71-89.

[26] Murasugi K., On the genus of the alternating knot, I, II, J. Math. Soc. Japan 10 (1958) 94-105, $235-248$.

[27] Murasugi K., On a certain subgroup of the group of an alternating link, Amer. J. Math. 85 (1963) $544-550$.

[28] OerTEL U., Homology branched surfaces: Thurston's norm on $H_{2}\left(M^{3}\right)$, in: Epstein D.B. (Ed.), Lowdimensional Topology and Kleinian Groups, Cambridge Univ. Press, 1986, pp. 253-272.

[29] Rolfsen D., Knots and Links, Publish or Perish, 1976.

[30] Stallings J., Constructions of fibred knots and links, in: Algebraic and Geometric Topology, Proc. Sympos. Pure Math., Vol. 32, American Mathematical Society, 1978, pp. 55-60.

[31] ThURston W.P., A norm for the homology of 3-manifolds, Mem. Amer. Math. Soc. 339 (1986) 99130.

[32] TURAEV V.G., The Alexander polynomial of a three-dimensional manifold, Math. USSR Sb. 26 (1975) 313-329.

[33] VIDUSSI S., The Alexander norm is smaller than the Thurston norm: a Seiberg-Witten proof, Preprint 99-6, École Polytechnique.

Curtis T. MCMullen

Mathematics Department,

Harvard University,

1 Oxford St,

Cambridge, MA 02138-2901, USA 